\title{
Selection rules for the orbital angular momentum of optically produced THz radiation
}

\author{
A. Curcio, ${ }^{1, *}$ S. Mou, ${ }^{2}$ L. Palumbo, ${ }^{2,3}$ S. Lupi,,${ }^{2,4}$ and M. Petrarca ${ }^{2,3}$ (1) \\ ${ }^{1}$ Centro de Laseres Pulsados (CLPU), Edificio M5. Parque Científico. C/Adaja, 8. 37185 Villamayor, Salamanca, Spain \\ 'INFN Roma1, Rome, Italy \\ ${ }^{3}$ S.B.A.I. Department, Roma University "La Sapienza”, Rome, Italy \\ ${ }^{4}$ Physics Department, Roma University "La Sapienza”, Rome, Italy \\ ${ }^{*}$ Corresponding author: acurcio@clpu.es
}

Received 8 December 2020; revised 18 February 2021; accepted 22 February 2021; posted 22 February 2021 (Doc. ID 416814 ); published 17 March 2021

In this work, we theoretically study the transduction of orbital angular momentum (OAM) $l$ for infrared pump lasers into the $\mathrm{THz}$ domain. In the case of optical rectification, the transduction of OAM occurs only through a spin-orbit interaction, with the selection rule on the OAM $l=0$ valid for any kind of polarization of the pump, which means that there is no transfer of OAM along the propagation axis. In difference frequency generation, the selection rule for the difference $\Delta l$ between the OAM of the pump fields with linear or circular polarization is $l=\Delta l$, whereas $l$ ranges from $\Delta l-2$ to $\Delta l+2$ in cases of both radial and azimuthal polarization. Moreover, for $\mathrm{THz}$ generation in the latter case, the high diffraction obtained with tightly focused pumps yields $l$ tending to $\Delta l \pm 2$, while $l$ tends to zero in the opposite case of large pump beams. (c) 2021 Optical Society of America

https://doi.org/10.1364/OL.416814

Orbital angular momentum (OAM) laser pulses [1,2] have generated much interest in the scientific community due to the wide range of interdisciplinary applications in which they can be used, such as spectroscopy $[3,4]$, telecommunication [5], and particle acceleration [6,7]. Within the same fields of application, $\mathrm{THz}$ pulses are increasingly becoming of scientific interest [8-12]. In this Letter, we derive the selection rules for the transduction of OAM during optical rectification (OR) and difference frequency generation (DFG) processes $[13,14]$ from infrared to $\mathrm{THz}$. Moreover, this work can be considered an extension of pulse shaping theories [15-17].

We highlight that the OAM transfer is affected by spin-orbit and orbit-orbit interactions between the pump and the polarization field, and we determine the effects of diffraction on the final expectation value of the OAM of the THz field.

The solution to the Maxwell equations for the magnetic field corresponding to nonlinear source terms, related to second-order polarization currents, is

$H_{\mathrm{NL}}^{u}(\vec{r}, \omega) \simeq \epsilon^{\mathrm{uqi}} \nabla_{q} \int_{V_{R}} \sigma_{i j k}^{(2)}(\omega) \frac{e^{i \frac{n(\omega) \omega}{c}\left|\vec{r}-\vec{r}^{\prime}\right|}}{4 \pi\left|\vec{r}-\vec{r}^{\prime}\right|}\left(E_{1}^{j} \star E_{2}^{k}\right)\left(\vec{r}^{\prime}, \omega\right) \mathrm{d}^{3} r^{\prime}$, where $\epsilon^{\text {uqi }}$ is the Levi-Civita tensor. The convolution product is denoted by $\star$. The Einstein summation convention is used for indices $(q, i)$ and $(j, k)$, the radiator volume is $V_{R}, n(\omega)$ is the linear refraction index evaluated at frequency $\omega, c$ is the speed of light in vacuum, and $\sigma^{(2)}$ is the second-order electric conductivity. The convolution between two external pump fields $E_{1,2}$ appearing in Eq. (1) is calculated assuming a general form for Gaussian pulses [18]:

$$
\left(E_{1}^{j} \star E_{2}^{k}\right)(\vec{r}, \omega)=\frac{2 \pi^{3 / 2} \tau_{1} \tau_{2}}{\sqrt{\tau_{1}^{2}+\tau_{2}^{2}}} E_{1}^{j}(\varrho, \varphi) E_{2}^{k}(\varrho, \varphi) e^{-\xi^{2}(z)},
$$

where $\varrho, \varphi, z$ are commonly defined cylindrical coordinates, and

$$
\begin{aligned}
\xi^{2}(z)= & \frac{\tau_{1}^{2} \tau_{2}^{2}(\omega-\Omega)^{2}}{\tau_{1}^{2}+\tau_{2}^{2}}-i\left(\frac{\tau_{2}^{2}}{v_{g 1}}+\frac{\tau_{1}^{2}}{v_{g 2}}\right) \frac{z(\omega-\Omega)}{\tau_{1}^{2}+\tau_{2}^{2}}+ \\
& -i K z+\left(\frac{1}{v_{g 1}}-\frac{1}{v_{g 2}}\right)^{2} \frac{z^{2}}{4\left(\tau_{1}^{2}+\tau_{2}^{2}\right)}-\frac{1}{2}\left(\alpha_{1}+\alpha_{2}\right) z
\end{aligned}
$$

We use $\tau_{x}$ for the rms pulse length, $\alpha_{x}$ for the linear absorption coefficient, and $v_{g x}$ for the group velocity of the $x$ field, where $x=1,2$ according to which pump field is considered. The angular frequency and wavenumber of the nonlinear polarization wave are $\Omega$ and $K$, respectively. To start, we develop our calculations focusing on the OR case, which is of great importance in the generation of $\mathrm{THz}$ radiation [19] from an infrared pump pulse $[15,18,20]$ interacting with a nonlinear crystal, for example, in the geometry shown in Fig. 1. For the OR process, the following conditions hold: $(\Omega, K)=(0,0), E_{1}=E_{0}^{*}$, $E_{2}=E_{0}, \quad \tau_{1}=\tau_{2}=\tau, \quad \alpha_{1}=\alpha_{2}=\alpha, \quad$ and $v_{g 1}=v_{g 2}=v_{g}$. Considering the far-field radiation emitted from the volume $V_{c}$ of the radiator crystal as OR radiation, the associated electric field is (via $\vec{E}=\hat{k} \times \mu_{0} c \vec{H}_{\mathrm{NL}}$ )

$$
\begin{aligned}
E_{i}(\vec{R}, \omega)= & -\frac{\omega^{2} n(\omega)}{4 \pi c^{2}} \frac{e^{i \frac{n(\omega) \omega}{c} R}}{R} \chi_{i j k}^{(2)}(\omega) \\
& \times \int_{V c} \mathrm{~d}^{3} r\left(E_{0}^{* j} \star E_{0}^{k}\right)(\vec{r}, \omega) e^{-i \vec{k} \cdot \vec{r}},
\end{aligned}
$$




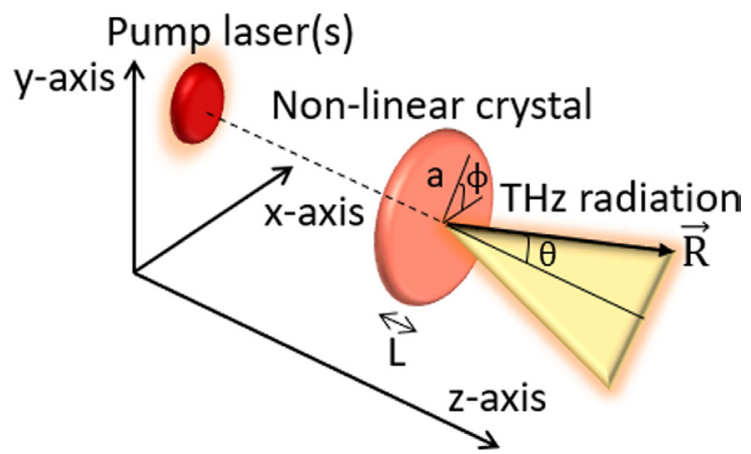

Fig. 1. Setup for optical generation of $\mathrm{THz}$ radiation and axes orientation considered in this Letter.

where $\vec{R}=R\{\cos \phi \sin \theta, \sin \phi \sin \theta, \cos \theta\}$ is the observation vector in spherical coordinates (Fig. 1). Notice also that $\vec{k}=\vec{R} n(\omega) \omega / c R, \chi_{i j k}^{(2)}$ is the second-order electric susceptibility tensor, and $\mu_{0}$ is the magnetic permeability in vacuum.

We consider a round crystal of radius $a$ and thickness $L$; then Eq. (4) can be processed in cylindrical coordinates as

$$
\begin{aligned}
E_{i}(\vec{R}, \omega)= & -\frac{\pi^{3 / 2} \omega^{2} n(\omega) \tau}{2 \sqrt{2} \pi c^{2}} \frac{e^{i \frac{n(\omega) \omega}{c} R}}{R} \int_{0}^{L} \mathrm{~d} z e^{-\xi^{2}(z)-i k z \cos \theta} \\
& \times \chi_{i j k}^{(2)}(\omega) \int_{0}^{2 \pi} \mathrm{d} \varphi \int_{0}^{a} \mathrm{~d} \varrho \varrho E_{0}^{* j}(\varrho, \varphi) E_{0}^{k}(\varrho, \varphi) \\
& \times e^{i k \varrho \sin \theta \cos (\phi-\varphi)},
\end{aligned}
$$

where $\varrho=\left(x^{2}+y^{2}\right)^{1 / 2}$, and $\varphi=\arctan (y / x)$. At this point, we analyze in detail the transverse and longitudinal integrals of Eq. (5). Concerning the transverse integral, we define

$D(\omega, \theta, \phi)=\int_{0}^{a} \mathrm{~d} \varrho \varrho \int_{0}^{2 \pi} \mathrm{d} \varphi E_{0}^{* j}(\varrho, \varphi) E_{0}^{k}(\varrho, \varphi) e^{i k \varrho \sin \theta \cos (\phi-\varphi)}$,

which is the Fraunhofer diffraction integral. We also define

$M(\omega, L)=\int_{0}^{L} \mathrm{~d} z e^{-\xi^{2}(z)-i k z \cos \theta}=-i e^{-\frac{\tau^{2} \omega^{2}}{2}}\left(\frac{1-e^{-i \kappa L}}{\kappa}\right)$,

which expresses a resonance related to the phase matching between the pump fields and the nonlinear polarization wave, where $\kappa=k \cos \theta+i \alpha-\omega / v_{g}$. An efficient radiation generation is obtained for those frequencies such that $\kappa \sim 0$, which is the phase matching condition for the OR process. This occurs (neglecting absorption) whenever the phase velocity of the OR wave $v_{\phi}=\omega / k \cos \theta$ equals the group velocity $v_{g}$ of the pump. The OR radiation will possess OAM if the expectation value of the operator $\hat{L}_{z}(\varphi)=-i \hbar \partial_{\phi}$ evaluated upon the field expressed by Eq. (4) is non-zero, i.e., $\left\langle\vec{E}\left|\hat{L}_{z}\right| \vec{E}\right\rangle \neq 0$. Indeed, the electric field can be interpreted as the wavefunction of the photon. Furthermore, for convenience, any component of the pump field can be expressed as a component of the corresponding Jones vector $\hat{e}$, i.e., $E_{0}^{j}=e^{j} E_{0}$. The photon-spin operators along three directions of space, $\hat{\sigma}_{x}, \hat{\sigma}_{y}, \hat{\sigma}_{z}$, where $z$ is the propagation direction and $x-y$ is the corresponding transverse plane, are represented by the following matrices:

$$
\hat{\sigma}_{z}=\hbar\left(\begin{array}{cc}
0 & -i \\
i & 0
\end{array}\right) \hat{\sigma}_{y}=\hbar\left(\begin{array}{ll}
0 & 1 \\
1 & 0
\end{array}\right) \hat{\sigma}_{x}=\hbar\left(\begin{array}{cc}
1 & 0 \\
0 & -1
\end{array}\right) .
$$

The eigenstate for a positive photon spin along $z$ is given by $\vec{s}_{1}=2^{-1 / 2}(1, i)^{T}$, while the eigenstate for the negative spin is $\vec{s}_{-1}=2^{-1 / 2}(1,-i)^{T}$, where $T$ stands for transpose. Indeed, $\vec{s}_{1}^{\dagger} \vec{s}_{1}=\left\langle\vec{s}_{1} \mid \vec{s}_{1}\right\rangle=1, \vec{s}_{-1}^{\dagger} \vec{s}_{-1}=\left\langle\vec{s}_{-1} \mid \vec{s}_{-1}\right\rangle=1$, and

$$
\begin{aligned}
\hat{\sigma}_{z} \vec{s}_{1} & =\frac{\hbar}{\sqrt{2}}\left(\begin{array}{cc}
0 & -i \\
i & 0
\end{array}\right)\left(\begin{array}{l}
1 \\
i
\end{array}\right)=\frac{\hbar}{\sqrt{2}}\left(\begin{array}{l}
1 \\
i
\end{array}\right)=\hbar \vec{s}_{1}, \\
\hat{\sigma}_{z} \vec{s}_{-1} & =\frac{\hbar}{\sqrt{2}}\left(\begin{array}{cc}
0 & -i \\
i & 0
\end{array}\right)\left(\begin{array}{c}
1 \\
-i
\end{array}\right)=-\frac{\hbar}{\sqrt{2}}\left(\begin{array}{c}
1 \\
-i
\end{array}\right)=-\hbar \vec{s}_{-1} .
\end{aligned}
$$

In terms of spin eigenstates of the photon, the Jones vectors [21] for different transverse polarization states can be expressed as

$$
\begin{aligned}
\hat{e}_{l} & =\left(\begin{array}{cc}
\cos \varphi_{0} & 0 \\
0 & \sin \varphi_{0}
\end{array}\right) \frac{\left(\vec{s}_{1}(1-i)+(1+i) \vec{s}_{-1}\right)}{2} \\
& =\left(\begin{array}{c}
\cos \varphi_{0} \\
\sin \varphi_{0}
\end{array}\right) \quad \text { linear, } \\
\hat{e}_{c} & =\vec{s}_{ \pm 1}=\frac{1}{\sqrt{2}}\left(\begin{array}{c}
1 \\
\pm i
\end{array}\right) \quad \text { right }(+) / \text { left }(-) \text { handed circular, } \\
\hat{e}_{r} & =\left(\begin{array}{cc}
\cos \varphi & 0 \\
0 & \sin \varphi
\end{array}\right) \frac{\left(\vec{s}_{1}(1-i)+(1+i) \vec{s}_{-1}\right)}{2} \\
& =\left(\begin{array}{c}
\cos \varphi \\
\sin \varphi
\end{array}\right) \quad \text { radial, } \\
\hat{e}_{\varphi} & =\left(\begin{array}{cc}
0-1 \\
1 & 0
\end{array}\right) \hat{e}_{r}=\left(\begin{array}{c}
-\sin \varphi \\
\cos \varphi
\end{array}\right) \quad \text { azimuthal, }
\end{aligned}
$$

where the first component of the Jones vector is along $x$ and the second along $y$. The angle $\varphi_{0}$ is constant and defines the polarization direction in the $x-y$ plane for a linear polarization state. The expectation value of $\hat{\sigma}_{z}$ over the polarization states listed above is calculated as $\left\langle\hat{e}_{l}\left|\hat{\sigma}_{z}\right| \hat{e}_{l}\right\rangle=0,\left\langle\hat{e}_{c}\left|\hat{\sigma}_{z}\right| \hat{e}_{c}\right\rangle= \pm \hbar$, and $\left\langle\hat{e}_{r}\left|\hat{\sigma}_{z}\right| \hat{e}_{r}\right\rangle=0$. It is possible to notice that only the circular polarization state carries photon spin along the propagation axis. The radial polarization state possesses spin components along the $y$ direction, i.e., $\left\langle\hat{e}_{r}\left|\hat{\sigma}_{y}\right| \hat{e}_{r}\right\rangle=2 \hbar \cos \phi \sin \phi$, and along the $x$ direction, i.e., $\left\langle\hat{e}_{r}\left|\hat{\sigma}_{x}\right| \hat{e}_{r}\right\rangle=\hbar\left(\cos ^{2} \phi-\sin ^{2} \phi\right)$ (note that $\left(\left\langle\hat{e}_{r}\left|\hat{\sigma}_{x}\right| \hat{e}_{r}\right\rangle^{2}+\left\langle\hat{e}_{r}\left|\hat{\sigma}_{y}\right| \hat{e}_{r}\right\rangle^{2}\right)^{1 / 2}=\hbar$, i.e., the modulus of the photon spin is preserved, as it must be, since it is an intrinsic property of the particle). For completeness, we mention that the photon spin for a linear polarization state forms an angle with the $x$ axis $\phi_{s}=\arctan \left(2 \cos \varphi_{0} \sin \varphi_{0} / \cos ^{2} \varphi_{0}-\sin ^{2} \varphi_{0}\right)$.

Let us now assume a collimated Laguerre-Gauss (LG) pump in cylindrical coordinates with beam size $w_{0}$, carrying an OAM $L_{z}=l_{0} \hbar$ ( $l_{0}$ is known as topological charge) [22,23]:

$$
\begin{aligned}
\vec{E}_{0}(\varrho, \varphi, z, t)= & E_{0} \hat{e}(\varphi)\left[\frac{\sqrt{2} \varrho}{w_{0}}\right]^{l_{0}} L_{p}^{l_{0}}\left[\frac{2 \varrho^{2}}{w_{0}^{2}}\right] \\
& \times e^{-\frac{\varrho^{2}}{w_{0}^{2}}-\frac{\left(t-z / v_{g}\right)^{2}}{4 \tau^{2}}-i l_{0} \phi+i\left(k_{0} z-\omega_{0} t\right)},
\end{aligned}
$$


where $p \geq 0$ is the radial index [24]. The angular frequency and the wavenumber of the pump wave are $\omega_{0}$ and $k_{0}$, respectively. The energy and OAM exchange between the pump field and the nonlinear crystal during OR occurs in such a way that

$$
\hat{L}_{z} \vec{E} \propto \int_{0}^{a} \mathrm{~d} \varrho \varrho E_{0}^{2}(\varrho) \hat{L}_{z}(\phi) \int_{0}^{2 \pi} \mathrm{d} \varphi e^{j}(\varphi) e^{k}(\varphi) e^{i k \varrho \sin \theta \cos (\phi-\varphi)}
$$

therefore, the OAM of the OR radiation field is determined by a spin-orbit interaction $[25,26]$, since $\hat{L}_{z}$ couples to the photonspin eigenstates.

To find the selection rules for the exchange of OAM during OR processes, the following integral identity turns out to be helpful:

$$
\int_{0}^{2 \pi} \mathrm{d} \varphi e^{i k \varrho \sin \theta \cos (\phi-\varphi)} e^{-i l_{0} \varphi}=2 \pi i^{l_{0}} J_{l_{0}}(k \varrho \sin \theta) e^{-i l_{0} \phi},
$$

where $J_{n}(x)$ is the $n$ th-order Bessel function of the first kind. Concerning linear and circular polarization of the pump, Eq. (13) allows us to demonstrate that OAM is not transferred along the $z$ direction, i.e., if we denote OAM of the OR field as $l$, then the selection rule for linear and circular polarizations is $l=0$. For radial and azimuthal polarizations, depending on the considered nonlinear crystal, the nonlinear susceptibility tensor can give rise to nonlinear polarization terms in Eq. (12) of three kinds: $e^{j}(\varphi) e^{k}(\varphi)=\cos ^{2} \varphi, \sin ^{2} \varphi, \pm \cos \varphi \sin \varphi$. The solution to Eq. (13) for any of these possible terms is

$$
\begin{aligned}
& \int_{0}^{2 \pi} \mathrm{d} \varphi e^{i k \varrho \sin \theta \cos (\phi-\varphi)} \cos ^{2} \varphi \\
& =\frac{\pi}{2}\left[2 J_{0}(k \varrho \sin \theta)-J_{-2}(k \varrho \sin \theta) e^{2 i \phi}-J_{2}(k \varrho \sin \theta) e^{-2 i \phi}\right],
\end{aligned}
$$

$$
\begin{aligned}
& \int_{0}^{2 \pi} \mathrm{d} \varphi e^{i k \varrho \sin \theta \sin (\phi-\varphi)} \sin ^{2} \varphi \\
& =\frac{\pi}{2}\left[2 J_{0}(k \varrho \sin \theta)+J_{-2}(k \varrho \sin \theta) e^{2 i \phi}+J_{2}(k \varrho \sin \theta) e^{-2 i \phi}\right],
\end{aligned}
$$

$$
\begin{aligned}
& \pm \int_{0}^{2 \pi} \mathrm{d} \varphi e^{i k \varrho \sin \theta \sin (\phi-\varphi)} \cos \varphi \sin \varphi \\
& \quad= \pm \frac{i \pi}{2}\left[J_{-2}(k \varrho \sin \theta) e^{2 i \phi}-J_{2}(k \varrho \sin \theta) e^{-2 i \phi}\right]
\end{aligned}
$$

The final state of the OR field is in general a superposition of $\hat{L}_{z}$ eigenstates carrying OAM $l=0,-2,2$, with an expectation value $\hbar^{-1}\left\langle\vec{E}\left|\hat{L}_{z}\right| \vec{E}\right\rangle=0$. In fact, the OR field is a superposition of the $\hat{L}_{z}$ eigenstate $l=0$ with a sub-superposition of eigenstates $l=-2$ and $l=+2$ with equal amplitudes. This can be verified by directly applying $\hat{L}_{z} \vec{E}$ as in Eq. (12) and using Eqs. (14)-(16). With the same theoretical description, we now demonstrate that via the DFG process, it is possible to transfer OAM along the $z$ direction. Let us choose two LG modes of the kind as Eq. (11) with amplitudes $E_{1}$ and $E_{2}$, respectively, corresponding to OAM $l_{1}$ and $l_{2}$. Then the integrand of Eq. (6) is modified by the term $E_{1}^{* j}(\varrho, \varphi) E_{2}^{k}(\varrho, \varphi)$, and the selection rules for linear and circular polarizations become $l=l_{2}-l_{1}=\Delta l$, in virtue of Eq. (13). For the DFG case, Eqs. (14)-(16) are generalized to

$$
\begin{gathered}
\int_{0}^{2 \pi} \mathrm{d} \varphi e^{i k \varrho \sin \theta \cos (\phi-\varphi)} e^{-i \Delta l \varphi} \cos ^{2} \varphi \\
=\frac{\pi}{2}\left[2 i^{\Delta l} J_{\Delta l}(k \varrho \sin \theta) e^{-i \Delta l \phi}\right. \\
+i^{\Delta l-2} J_{\Delta l-2}(k \varrho \sin \theta) e^{i(-\Delta l+2) \phi} \\
\left.+i^{\Delta l+2} J_{\Delta l+2}(k \varrho \sin \theta) e^{i(-\Delta l-2) \phi}\right] \\
\int_{0}^{2 \pi} \mathrm{d} \varphi e^{i k \varrho \sin \theta \sin (\phi-\varphi)} e^{-i \Delta l \varphi} \sin { }^{2} \varphi \\
=\frac{\pi}{2}\left[2 i^{\Delta l} J_{\Delta l}(k \varrho \sin \theta) e^{-i \Delta l \phi}-i^{\Delta l-2} J_{\Delta l-2}(k \varrho \sin \theta)\right. \\
\left.\times e^{i(-\Delta l+2) \phi}+-i^{\Delta l+2} J_{\Delta l+2}(k \varrho \sin \theta) e^{i(-\Delta l-2) \phi}\right] \\
\pm \int_{0}^{2 \pi} \mathrm{d} \varphi e^{i k \varrho \sin \theta \sin (\phi-\varphi)} e^{-i \Delta l \varphi} \cos \varphi \sin \varphi \\
= \pm \frac{\pi}{2}\left[i^{\Delta l-1} J_{\Delta l-2}(k \varrho \sin \theta) e^{i(-\Delta l+2) \phi}+\right. \\
\left.\quad-3 J_{\Delta l+2}(k \varrho \sin \theta) e^{i(-\Delta l-2) \phi}\right]
\end{gathered}
$$

Thus, in the DFG process, for radial and azimuthal polarizations, the final state of the radiation field will be a superposition of $\hat{L}_{z}$ eigenstates carrying OAM $\Delta l, \Delta l-2, \Delta l+2$, with amplitudes depending on the radiation spectrum through the Fraunhofer diffraction integral and with an expectation value $\hbar^{-1}\left\langle\vec{E}\left|\hat{L}_{z}\right| \vec{E}\right\rangle$ ranging from $\Delta l-2$ to $\Delta l+2$. The presence of \pm 2 in the expectation value of $\hat{L}_{z}$ is related to a spin-orbit interaction that couples the spins of the two pump photons involved in the DFG to the OAM of the radiation field. The presence of $\Delta l$ instead is justified as an orbit-orbit interaction, differently with respect to the OR case. It is interesting to note the general property that when the condition $w_{0} \gg 1 / k$ is met, i.e., very large pump beams, then $J_{\Delta l-2}(k \varrho \sin \theta) \sim J_{\Delta l+2}(k \varrho \sin \theta)$, and the expectation value of $\hat{L}_{z}$ tends to zero. On the contrary, in the highly diffracting case, i.e., $w_{0} \ll 1 / k$, the expectation value of $\hat{L}_{z}$ tends to $\Delta l-2$ for $\Delta l \gg 1$, and to $\Delta l+2$ for $\Delta l \ll-1$. In fact, within the integration domain, these asymptotic behaviors for $\Delta l \gg 1$ and $\Delta l \ll-1$ are, respectively, related to the fact that $J_{\Delta l-2}(k \varrho \sin \theta) \gg J_{\Delta l}(k \varrho \sin \theta) \gg J_{\Delta l+2}(k \varrho \sin \theta)$, and $J_{\Delta l+2}(k \varrho \sin \theta) \gg J_{\Delta l}(k \varrho \sin \theta) \gg J_{\Delta l-2}(k \varrho \sin \theta)$.

Let us now consider the general case of the DFG process triggered by two radially polarized LG modes propagating in a diethylaminosulfur trifluoride crystal $(a=5 \mathrm{~mm}, L=1 \mathrm{~mm})$. The modes have the following characteristics: $p=1$, $w_{0}=1 \mathrm{~mm}, \quad \tau=1 \mathrm{ps}, \quad \omega_{01}=982 \mathrm{THz}, \quad \omega_{02}=995 \mathrm{THz}$, $l_{1}=0$, and $l_{2}=1$. The crystal properties are $\chi_{111}^{(2)}=210 \mathrm{pm} / \mathrm{V}$, $\chi_{122}^{(2)}=32 \mathrm{pm} / \mathrm{V}, \quad \chi_{212}^{(2)}=\chi_{221}^{(2)}=25 \mathrm{pm} / \mathrm{V}$, when pumped at $1907 \mathrm{~nm}$ [27]. In this configuration, the DFG field is a superposition of $\hat{L}_{z}$ eigenstates carrying OAM $1,-1,3$. The expectation values for the horizontal and vertical components 

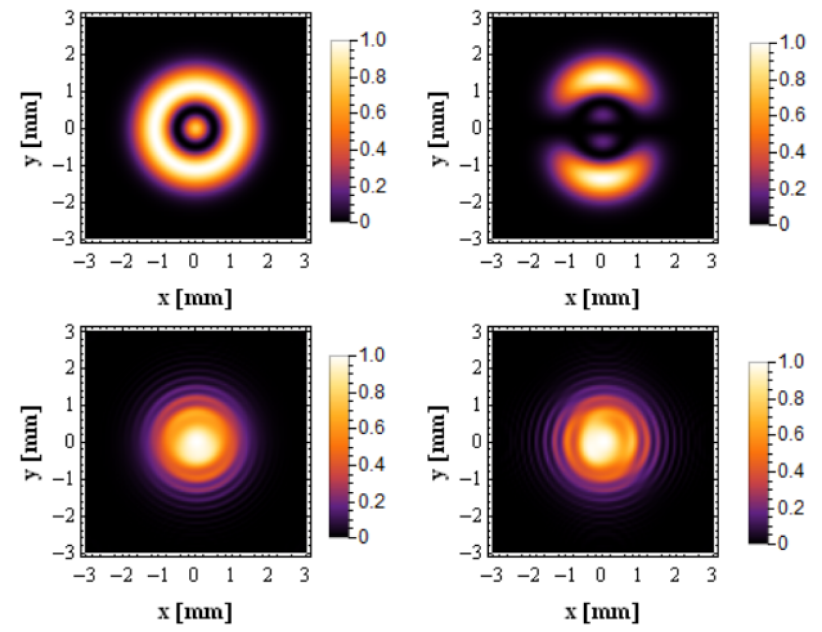

Fig. 2. Intensity profiles (arbitrary units). First row: on the left, first pump; on the right, second pump. Second row: interference of the horizontal (left) and vertical (right) components of the DFG field with a plane wave, observed at $10 \mathrm{~cm}$ from the crystal and at frequency $\Delta \omega_{0}=\omega_{02}-\omega_{01}$.

of the DFG field are, respectively, $\left\langle E_{x}\left|\hat{L}_{z}\right| E_{x}\right\rangle \simeq 0.79 \hbar$ and $\left\langle E_{y}\left|\hat{L}_{z}\right| E_{y}\right\rangle \simeq-0.66 \hbar$.

In Fig. 2, we show a comparison among the intensity transverse profiles relative to the vortex beams under consideration. The DFG fields are calculated by Eq. (5) (expressed for the DFG case) combined with Eqs. (17)-(19), while also considering the Fresnel coefficient for the transmission at the crystal-vacuum interface. The images of interference are computed by adding the Gaussian field $E_{g}=\mathcal{E}_{0} \exp \left[-\left(x^{2}+y^{2}\right) / 2 w_{0}^{2}-i \psi_{0}\right]$ and then considering the modulus square $\left|E_{g}+E_{i}\right|^{2}$, with the field amplitude $\mathcal{E}_{0}$ adjusted to $3 / 1000$ of the DFG field amplitude and $\psi_{0}=\pi / 7$ arbitrarily chosen for yielding a realistic interference pattern on the virtual screen. An important remark is that Eq. (5) considers parallel pump beams, resulting in a nondependence of the expected value of the OAM upon the crystal length.

The authors of Refs. $[28,29]$ demonstrate that in their DFG experimental conditions, the transduction of OAM occurs under the conservation of OAM. Concerning this Letter, we have found that it is related to the conservation of the total angular momentum; therefore, our result is a generalization in this sense, because it does not apply only to the case of linear polarization of the pump. The selection rules we have established are in agreement with Refs. $[28,29]$ for the DFG case and also in agreement with Refs. [30,31] for the OR case, where it has been experimentally demonstrated that, unless further modifications to the optical system are performed, the expectation value for the OAM of the OR field is zero.

In conclusion, we have developed an analytical theory to describe the transduction of OAM in DFG and OR processes with particular regard to $\mathrm{THz}$ generation. For OR, the transduction of OAM occurs only through a spin-orbit interaction with $l=0$ for any kind of polarization states of the pump beams (no transfer of OAM along the propagation axis). For DFG, the selection rule for the difference $\Delta l$ between the OAM of the pump fields with linear or circular polarization is $l=\Delta l$, whereas $l$ ranges from $\Delta l-2$ to $\Delta l+2$ in the case of radial and azimuthal polarizations. Moreover, for $\mathrm{THz}$ generation in the latter cases, the high diffraction obtained with tightly focused pumps yields $l$ tending to $\Delta l \pm 2$, while $l$ tends to zero for large pump beams.

Disclosures. The authors declare no conflicts of interest.

\section{REFERENCES}

1. M. Padgett, J. Courtial, and L. Allen, Phys. Today 57(5), 35 (2004).

2. A. Mair, A. Vaziri, G. Weihs, and A. Zeilinger, Nature 412, 313 (2001).

3. M. A. Noyan and J. M. Kikkawa, Appl. Phys. Lett. 107, 032406 (2015).

4. A. D'Arco, L. Tomarchio, V. Dolci, P. Di Pietro, A. Perucchi, S. Mou, M. Petrarca, and S. Lupi, Condens. Matter 5, 47 (2020).

5. A. E. Willner, H. Huang, Y. Yan, Y. Ren, N. Ahmed, G. Xie, C. Bao, L. Li, Y. Cao, Z. Zhao, J. Wang, M. P. J. Lavery, M. Tur, S. Ramachandran, A. F. Molisch, N. Ashrafi, and S. Ashrafi, Adv. Opt. Photon. 7, 66 (2015).

6. J. Mendonça and J. Vieira, Phys. Plasmas 21, 033107 (2014).

7. A. Curcio, A. Marocchino, V. Dolci, S. Lupi, and M. Petrarca, Sci. Rep. 8, 1052 (2018).

8. T. Kampfrath, A. Sell, G. Klatt, A. Pashkin, S. Mährlein, T. Dekorsy, M. Wolf, M. Fiebig, A. Leitenstorfer, and R. Huber, Nat. Photonics 5, 31 (2011).

9. Z. Jin, A. Tkach, F. Casper, V. Spetter, H. Grimm, A. Thomas, T. Kampfrath, M. Bonn, M. Kläui, and D. Turchinovich, Nat. Phys. 11, 761 (2015).

10. S. W. Jolly, N. H. Matlis, F. Ahr, V. Leroux, T. Eichner, A.-L. Calendron, H. Ishizuki, T. Taira, F. X. Kärtner, and A. R. Maier, Nat. Commun. 10, 2591 (2019).

11. D. Zhang, A. Fallahi, M. Hemmer, X. Wu, M. Fakhari, Y. Hua, H. Cankaya, A.-L. Calendron, L. E. Zapata, N. H. Matlis, and F. X. Kärtner, Nat. Photonics 12, 336 (2018).

12. M. Di Fabrizio, A. D’Arco, S. Mou, L. Palumbo, M. Petrarca, and S. Lupi, Appl. Sci. 11, 562 (2021).

13. R. W. Boyd, Nonlinear Optics (Academic, 2019).

14. K. Furuki, M.-T. Horikawa, A. Ogawa, K. Miyamoto, and T. Omatsu, Opt. Express 22, 26351 (2014).

15. A. Curcio, V. Dolci, S. Lupi, and M. Petrarca, Opt. Lett. 43, 783 (2018).

16. S. Cialdi, M. Petrarca, and C. Vicario, Opt. Lett. 31, 2885 (2006).

17. S. Cialdi, C. Vicario, M. Petrarca, and P. Musumeci, Appl. Opt. 46, 4959 (2007).

18. A. Curcio and M. Petrarca, Opt. Lett. 45, 1619 (2020).

19. P. Rasekh, M. Saliminabi, M. Yildirim, R. W. Boyd, J.-M. Ménard, and K. Dolgaleva, Opt. Express 28, 3237 (2020).

20. A. Curcio and M. Petrarca, Opt. Lett. 44, 1011 (2019).

21. R. C. Jones, J. Opt. Soc. Am. 31, 488 (1941).

22. R. A. Beth, Phys. Rev. 48, 471 (1935).

23. L. Allen, M. W. Beijersbergen, R. Spreeuw, and J. Woerdman, Phys. Rev. A 45, 8185 (1992).

24. M. Abramowitz, I. A. Stegun, and R. H. Romer, Handbook of Mathematical Functions with Formulas, Graphs, and Mathematical Tables (1988).

25. K. Y. Bliokh, F. J. Rodrguez-Fortuño, F. Nori, and A. V. Zayats, Nat. Photonics 9, 796 (2015).

26. H.-J. Wu, H.-R. Yang, C. Rosales-Guzmán, W. Gao, B.-S. Shi, and Z.-H. Zhu, Phys. Rev. A 100, 053840 (2019).

27. M. Jazbinsek, U. Puc, A. Abina, and A. Zidansek, Appl. Sci. 9, 882 (2019).

28. K. Miyamoto, K. Sano, T. Miyakawa, H. Niinomi, K. Toyoda, A. Vallés, and T. Omatsu, Opt. Express 27, 31840 (2019).

29. A. Camper, H. Park, Y. H. Lai, H. Kageyama, S. Li, B. K. Talbert, C. I. Blaga, P. Agostini, T. Ruchon, and L. F. DiMauro, Opt. Lett. 42, 3769 (2017).

30. K. Miyamoto, B. J. Kang, W. T. Kim, Y. Sasaki, H. Niinomi, K. Suizu, F. Rotermund, and T. Omatsu, Sci. Rep. 6, 38880 (2016).

31. A. Al Dhaybi, J. Degert, E. Brasselet, E. Abraham, and E. Freysz, J. Opt. Soc. Am. B 36, 12 (2019). 